\title{
Fast Linear Elastic Matching Without Landmarks
}

\author{
Samson J. Timoner ${ }^{1}$, W. Eric L. Grimson ${ }^{1}$, Ron Kikinis ${ }^{2}$, and \\ William M. Wells $\mathrm{III}^{1,2}$ \\ 1 MIT AI Laboratory, Cambridge MA 02139, USA \\ samson@ai.mit.edu \\ 2 Brigham and Women's Hospital, Harvard Medical School, Boston MA, USA
}

Introduction: Non-rigid matchers generally constrain estimated displacement fields in uniform intensity regions of medical data. Elastic models are popular regularizers since they are easy to understand and simulate, and their smoothness properties may be as likely as other constraints.

Unfortunately, using an elastic energy function with image driving forces leads to ill-conditioned equations. To better constrain these equations, other authors have added landmarks or otherwise changed the boundary conditions of the problem[1]. However, reliable landmarks are difficult to find in medical images and we therefore avoid them. Our main contribution in this paper is to develop a fast nonrigid registration method using the ill-conditioned equations.

Methods: The matching process starts by meshing one volume with tetrahedra. We have written a tetrahedral mesher that accurately and compactly represents often irregular medical shapes without creating skewed tetrahedra. We use a probabilistic framework to match the mesh to the other volume by maximizing the log probability of the deformation field given the data, $\log P(\boldsymbol{r} \mid$ data $)$. Equivalently, we maximize $\log P($ data $\mid \boldsymbol{r})+\log P(\boldsymbol{r})$.

Our image agreement term, $P($ data $\mid \boldsymbol{r})$, is based on the joint probability $P\left(I_{2}, I_{1}\right)$ that the volumes have joint intensities $\left(I_{2}, I_{1}\right)$ when registered. Many authors assume the intensity distribution of each voxel is independent. A continuous version of this idea is more suitable to volumetric representations. Using this assumption, $\log P($ data $\mid \boldsymbol{r})=\sum_{\text {Tetrahedra }} \int_{\text {tet }} \log P\left(I_{2}, I_{1}\right) d V$, where $I_{2}$ changes depending on the warp.

The probability of a deformation, $P(\boldsymbol{r})$, can be estimated using statistical physics where the probability that a system is in a configuration is $e^{-E / T}$ where $T$ is a temperature and we choose $E$ as the linear elastic energy of the mesh. That energy can be locally written as is $\frac{1}{2} \boldsymbol{r}^{T} K \boldsymbol{r}$ where $K$ is an elasticity matrix[2].

We use a modified Newton based solver to find the zeros in the gradient of the maximization function. Given the guess $\boldsymbol{r}_{\boldsymbol{k}}$ in the $k^{t h}$ iteration, the change in the deformation field, $\boldsymbol{\delta} \boldsymbol{r}$, in the $k+1^{\text {th }}$ iteration solves

$$
\left[\frac{K}{T}+\epsilon I\right] \boldsymbol{\delta} \boldsymbol{r}=\frac{-K \boldsymbol{r}_{\boldsymbol{k}}}{T}+\sum_{\text {Tetrahedra }} \frac{d\left(\int_{t e t} \log P\left(I_{2}, I_{1}\right) d V\right)}{d \boldsymbol{r}} .
$$


Because $K$ is ill conditioned [1], we use a standard method to improve the matrix conditioning by adding a small $\epsilon$ to the diagonal of the matrix.

After the addition of this term, the elasticity matrix is still poorly-conditioned. Solving a poorly-conditioned linear system is possible using standard Krylov subspace based solvers, though may require many iterations. One common method in a Newton solve is to find an approximate solution to the matrix equation, using only a few iterations of the iterative matrix solve. Using this technique, the matrix solve time can be improved by more that a factor of 100 . And, because there may be no exact solution, little is lost in solving the system approximately.

Experiments: We tested our matcher applied to a the manually segmented, left hippocampus-amygdala complex from a data set of 30 patients. One complex from a randomly chosen patient was meshed and matched with the rest of the patients. These data sets represent an interesting challenge because of the need of the bulbous head of one amygdala to be captured by the other (Figure 1).

For 1000 node meshes the matcher converged in under 50 iterations in less than 1 minute on a $450 \mathrm{Mhz}$ Pentium III. Tests on larger meshes showed that 50 iterations were still sufficient and, as expected, the iteration time scaled linearly with the number of nodes. Figure 1 shows the error in the match. In the cases with highest error, we found the segmentations to have unphysical protrusions or minimal resemblance to the other data sets. The matcher tended to smooth these features leading to errors.
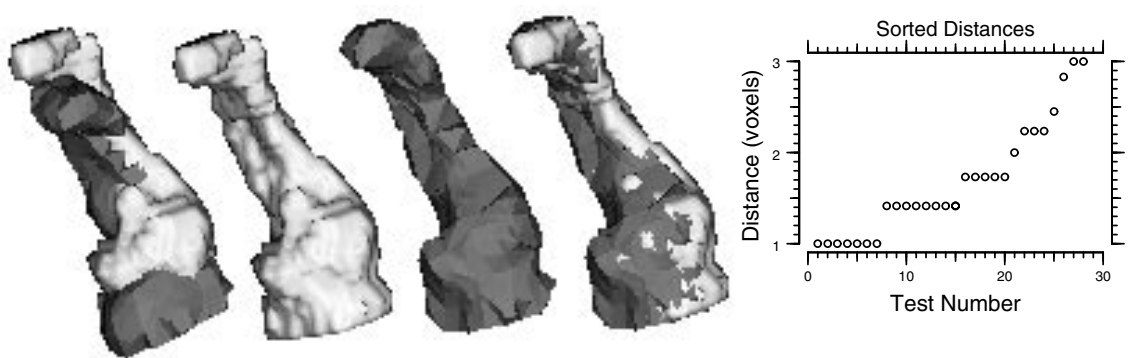

Fig. 1. The left image shows the initial alignment of the mesh (dark) with a triangulated surface of a labeled amygdala (light). The remaining images shows the surfaces after warping. The plot shows the distance between the two surfaces for each match in voxels, calculated as the upper quantile of the undirected Hausdorff metric.

Conclusions: We presented a fast, elastic volumetric matcher that does not use landmarks. We tested the matcher using label maps and showed good results.

Acknowledgements: S.J. Timoner is supported by the Fannie and John Hertz Foundation and NSF ERC grant, J.H.U Agreement \#8810274. W. Wells was supported by the same NSF grant. Dr. Martha Shenton provided segmented images for the matching experiments. Her work was supported by NIMH grants R01 50740 and K02 01110, and a Veterans Administration Merit Award. 


\section{References}

1. Y. Wang and L. H. Staib. Elastic model based non-rigid registration incorporating statistical shape information. In MICCAI 98, pages 1162-1173, Cambridge, MA, Oct. 1998.

2. O.C. Zenkiewicz and R.L. Taylor. The Finite Element Method, volume 1. McGrawHill, Berkshire, England, fourth edition, 1989. 